\title{
Organic Superconductors
}

\author{
I.F. Schegolev
}

\author{
Institute of Solid State Physics \\ USSR Academy of Sciences, Chernogolovka
}

The search for organic superconductors began some 20 years ago in the hope of creating materials with a nonphonon mechanism for superconductivity to permit one to break through the transition temperature barrier which was $25 \mathrm{~K}$ at the time.

Many hundreds (maybe thousands - who has counted them?) of new organic substances have been synthesized and in 1979, K. Bechgaard from the University of Copenhagen described a novel organic "metal", a salt consisting of an organic cation, tetramethyltetraselenofulvalenium, and an inorganic anion, $\mathrm{PF}_{6}$. D. Jérome and coworkers at the University of Paris-Sud, Orsay reported a year later that it passed into a superconducting state at temperatures near $1 \mathrm{~K}$ and pressures on the order of $10 \mathrm{kbar}$ [1]. This was therefore the first organic superconductor $[2$, $3]$. In the 10 years since then about 20 organic metals have been found to exhibit superconductivity at ambient pressure, and more than 15 at slightly elevated ones, with the maximum transition temperature reaching $15.5 \mathrm{~K}$.

\section{Structure}

All of the organic superconductors belong to the class of so-called ion-radical salts based on the following six organic molecules with similar constitutions (see Fig. 1):

tetramethyltetraselenofulvalen (TMTSF) (bisethylenditio)-tetrafiafulvalen

(BEDT-TTF, ET for short)

metal complexes of dimercaptoisotrition (M(dmit) $\left.)_{2}\right)$

dimethyl(ethylenditio)diselena-ditiafulvalen (DMET)

methylenditio-tetratiafulvalen (MDT-TTF) bis(ethylendioxy)-tetratiafulvalen (BEDO-TTF)

Professor I.F. Schegolev is an experimental physicist with the Institute of Solid State Physics, USSR Academy of Sciences, Moscow District, SU - 142432 Chernogolovka.
Fig. 1 - The six organic molecules which form the basis for the synthesis of organic superconductors.
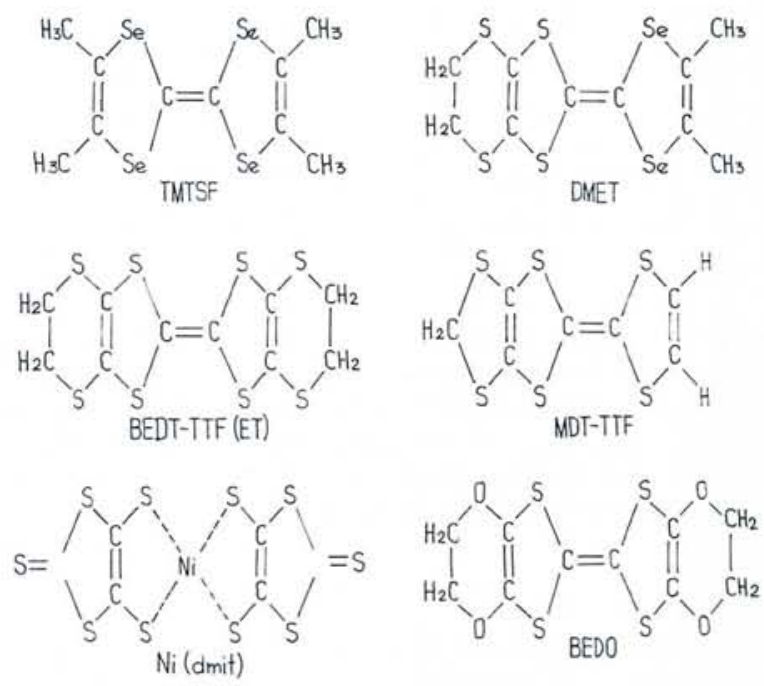

Fig. 2 - The crystal structure of the quasi one-dimensional Bechgaard salt with the general composition (TMTSF) ${ }_{2} X$.

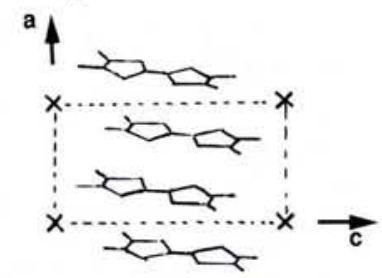

These molecules contain completely closed electron shells and form in themselves insulating molecular crystals. A general method for obtaining the organic metals consists in their partial oxidation (or reduction) by some organic or inorganic anions (or cations), giving rise to the formation of the salts with compositions of the type $(\mathrm{TMTSF})_{2}^{+}\left(\mathrm{ClO}_{4}\right)$;, $(\mathrm{ET})_{2}^{+} \mathrm{I}_{3}^{-}$and $\left[\mathrm{N}\left(\mathrm{CH}_{3}\right)_{4}\right]^{+}\left[\mathrm{Ni}(\mathrm{dmit})_{2}\right]_{2}^{-}$. In these compounds, an anion group takes one electron from (or a cation group gives up one electron to) each pair of organic molecules, transferring them into a state with the formal valence $1 / 2$. When packed into a crystal, these mixed valence states form a partially filled band of extended states (a conduction band) giving rise to a metallic type of electron system. The crystals exhibit highly anisotropic electronic properties because the conduction band of organic metals is primarily built up from

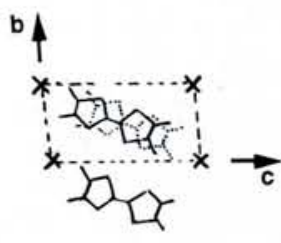

highly anisotropic electron-type wave functions.

\section{Quasi one-dimensional conductors}

Fig. 2 shows the crystal structure of a compound, with the general composition (TMTSF) ${ }_{2} \mathrm{X}$, that exemplifies a socalled Bechgaard salt. Each of these salts is to a first approximation a molecular crystal with intermolecular distances close to the Van der Waals' bond length. However, the spacings between TMTSF molecules in a stack along the $a$-direction are slightly shortened owing to partial overlap of the electrontype wave function. Much less overlap arises for molecules belonging to different stacks. Finally, there is almost no contact in the $c$-direction between TMTSF molecules in the different layers separated by layers of anions. We are as a result dealing here with quasi onedimensional metals having room tempe- 

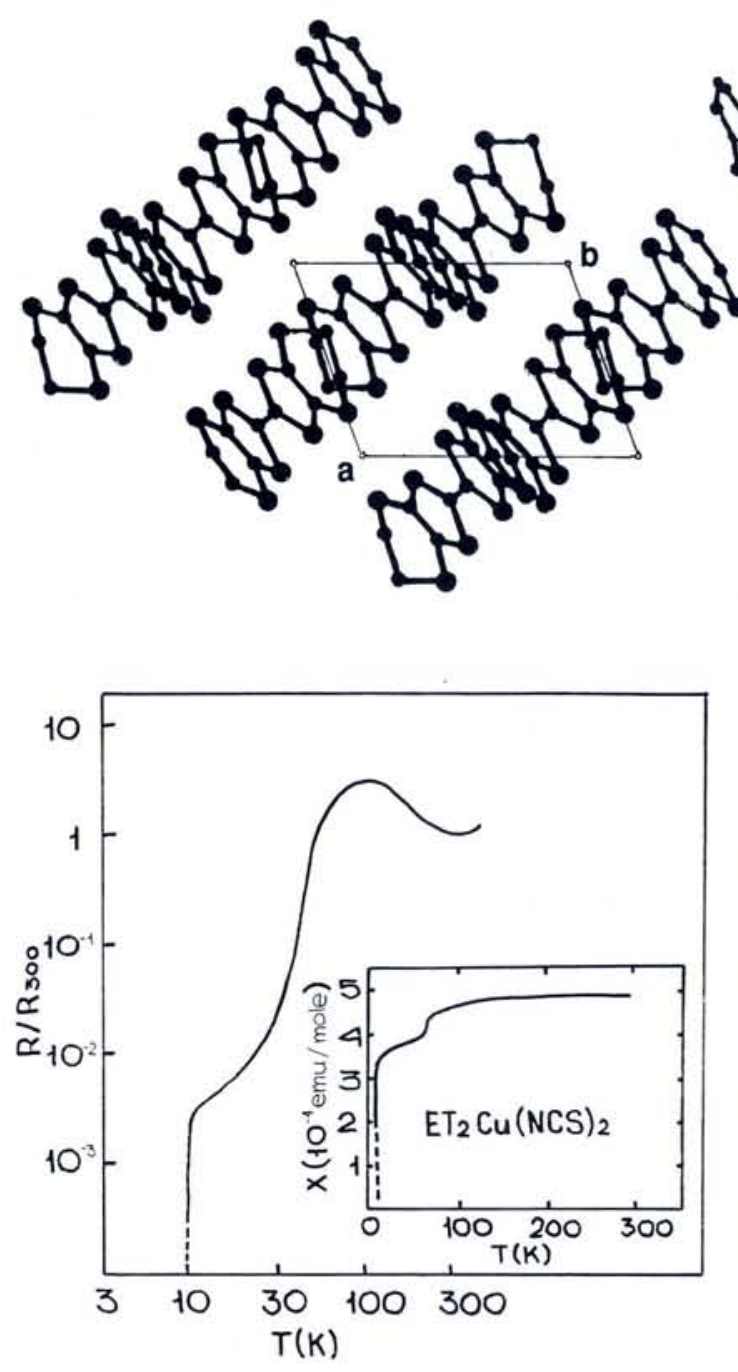

Fig. 4 - The temperature dependence of the resistivity of $(\mathrm{ET})_{2} \mathrm{Cu}(\mathrm{NCS})_{2}$. The resistivity, in exhibiting semiconducting behaviour, increases on cooling from $300 \mathrm{~K}$ to $100 \mathrm{~K}$. The insert showing the temperature dependence of the paramagnetic susceptibility reveals a small jump in the sus. ceptibility at $70 \mathrm{~K}$ accompanying the semiconductor-metal transition. The decrease in both the resistivity and the susceptibility at $10 \mathrm{~K}$ announces the superconducting transition. rature electrical conductivities in the $a$-, $b$ - and $c$-directions that are typically on the order of $5 \times 10^{2}, 1$ and $2 \times 10^{-3}$ $\mathrm{ohm}^{-1} \mathrm{~cm}^{-1}$, respectively.

\section{Quasi two-dimensional conductors}

In other classes of organic superconductors, the interstack interaction is more pronounced than in the Bechgaard salts resulting in a rather two-dimensional character. The crystal structure of a salt of this type, $\beta-(E T)_{2} I_{3}$, is illustrated in Fig. 3. Owing to the presence of eight sulfur atoms within the rings, many contacts of reduced length are formed among the stacks, thus giving rise to relatively high conductivities in all directions in the $a b$-plane: typical values are 30,20 and $5 \times 10^{-2} \mathrm{ohm}^{-1} \mathrm{~cm}^{-1}$ along the $a-b$ - and $c$-directions, respectively. This situation is characteristic of known organic superconductors except the Bechgaard salts. Anisotropy of the conductivity in the $a b$-plane of $\beta-(E T)_{2} I_{3}$ in fact seems to be slightly larger than $3: 2$ because the main directions of the conductivity tensor do not coincide with the crystal axes.

\section{Inherent Instabilities}

Quasi one-dimensional electron systems are characterized by an inherent instability towards charge or spin density wave formation (the Peierls instability) which competes with the superconducting instability. As a result, all known quasi one-dimensional metals (not necessarily organic) sooner or later pass into an insulating charge or spin density wave state on lowering the temperature. This behaviour is also a feature of all the Bechgaard salts except (TMTSF) ${ }_{2} \mathrm{ClO}_{4}$ where a low temperature structural transformation prevents the Peierls transition. This compound retains its metallic state on careful cooling and exhibits superconductivity at ambient pressure. It seems that a sufficiently slow cooling rate allows the noncentrosymmetric $\mathrm{ClO}_{4}^{-}$ions to order three dimensionally at low temperatures. The other Bechgaard salts need some pressure to enhance interchain coupling resulting in the suppression of all dielectric and magnetic instabilities and consequently stabilizing a superconducting ground state.
As is usually the case, the superconducting state can be suppressed by applying a magnetic field. What it is surprising is that a sufficiently strong field has been found to suppress not only the superconducting state but also the normal metallic state, by transforming the substance back into a spin density wave state with a low density of carriers. This phenomenon, the result of a field induced spin density wave, comes about because the strong magnetic field applied in the $c$-direction (see Fig. 2) restricts electron motion in the $b$-direction thereby making the system more one-dimensional. Metal-insulator transitions arise at certain field strengths, manifesting themselves in some peculiarities of the resistivity, Hall voltage, magnetic susceptibility, etc. The study of these and related phenomena has yielded much useful information concerning the properties of lowdimensional electron systems. In particular, the Hall resistance does not increase linearly with the applied magnetic field. It exhibits instead a quantized behaviour with plateaus of magnitude $\hbar / 2 n e^{2}$ ( $n=$ integer). This effect is reminiscent of the quantum hall effect of a two-dimensional electron gas, but the interpretation is different.

\section{Structural Modifications}

Quasi two-dimensional organic metals of the BEDT, M(dmit) ${ }_{2}$, DMET and MDT-TTF families do not exhibit the Peierls instability. However, they are all characterized by a specific structure lability which seems to be associated with the almost molecular character of their crystals. This lability first of all reveals itself by the existence of numerous polymorphic modifications of the same composition. There are, for example, $\alpha-\beta-, \nu$ - and $\theta$-phases of $(E T)_{2} \mathrm{I}_{3}$ distinguishable only by the type of molecular packing in the crystal and having different physical properties. These substance are also often characterized by many structural and electronic transformations that take place on lowering the temperature or increasing the applied pressure.

\section{$(\mathrm{ET})_{2} \mathrm{Cu}(\mathrm{NCS})_{2}$}

As a very interesting example I mention the metal with the composition $(\mathrm{ET})_{2} \mathrm{Cu}(\mathrm{NCS})_{2}$ which exhibits a superconducting transition temperature $T_{c}$ of $10.5 \mathrm{~K}$. As can be seen in Fig. 4, it behaves as a semiconductor in the temperature interval between 300 and $100 \mathrm{~K}$. With further cooling, the resistivity fall characteristic of a metal only begins below $90 \mathrm{~K}$, suggesting a rather unusual insulator-to-metal transition that 
occurs with a lowering of the temperature. This inference has been confirmed by the observation of a small jump in the magnetic susceptibility at $70 \mathrm{~K}$ (see the inset in Fig. 4). The nature of this unusual transformation remains unclear.

\section{$\beta-(E T)_{2} I_{3}$}

Another instructive example of a molecule showing complex structural transitions is given by $\beta-(E T)_{2} I_{3}$. It can exist in two different phases: There is a phase called $\beta_{L}$ which has some disorder associated with the random packing of peripheral ethylene groups in one or two possible configurations. The other phase $\beta_{H}$ is free of this $\mathrm{CH}_{2}$-group disorder and can only be stabilized at low temperatures provided cooling is performed following a special path in the $T$ - $P$ diagram, going around a critical point located at $p_{c}=0.5 \mathrm{kbar}$ and $T_{c}$ $180 \mathrm{~K}$. In spite of rather small differences in their structures and physical properties, the two phases are distinguished very markedly by their $T_{c}$ temperatures of $1.5 \mathrm{~K}$ and $\approx 8 \mathrm{~K}$ for $\beta_{\mathrm{L}}$ and $\beta_{\mathrm{H}^{\prime}}$, respectively.

\section{Normal State Electronic Properties}

The electrical resistivity $\rho$ of conventional metals is mainly determined by the scattering of electrons on phonons and impurities. The latter process predominantly manifests itself at low temperatures below the Debye temperature $T_{D}$ where there are almost no phonons present. It results in the appearance of a finite residual resistance $\rho_{0}$ as $T \rightarrow 0$ $K$. At high temperatures $\left(T>T_{D}\right)$ where all the phonons are equally excited and impurity scattering as a rule plays no rôle, the resistivity is proportional to the temperature. In the regime of intermediate temperatures, when both scattering mechanisms are acting, the resistivity decreases on cooling as

$$
\rho=\rho_{0}+a T^{n}
$$

with $n$ between 3 and 5 .

\section{Electron-electron scattering}

The resistivity behaviour of organic metals is quite different. In both the quasi one-dimensional Bechgaard salts and the quasi two-dimensional salts of BEDT-TTF and its derivatives, $\rho$ is roughly proportional to $T^{2}$ over a wide temperature range below room temperature. This quadratic temperature dependence has been found to hold exactly in some cases at low temperatures (see, for example, Fig. 5 for a number of BEDT-TTF salts at $T<20 \mathrm{~K}$ ). It can been understood as a manifestation of the fact that the main contribution to the resistivity comes from electron-
Fig. 4 - The temperature dependence of the resistivity of $(\mathrm{ET})_{2} \mathrm{Cu}(\mathrm{NCS})_{2}$. The resistivity, in exhibiting semiconducting behaviour, increases on cooling from $300 \mathrm{~K}$ to $100 \mathrm{~K}$. The insert showing the temperature dependence of the paramagnetic susceptibility reveals a small jump in the sus. ceptibility at $70 \mathrm{~K}$ accompanying the semiconductor-metal transition. The decrease in both the resistivity and the susceptibility at $10 \mathrm{~K}$ announces the superconducting transition.

electron scattering (this mechanism usually plays no rôle in ordinary metals). The scattering clearly becomes an essential feature of organic metals owing to the low-dimensional character of their electron systems.

Understanding is still needed of the many important quantitative details of the processes which magnify electronelectron scattering so strongly and to a level that, against background, the phonon contribution to the resistivity is completely invisible. In particular, the very large pressure dependence of the transport properties of Bechgaard salts which was noticed in the early studies of the Orsay group cannot be understood unless Coulombic interactions play a dominant rôle in the electronic properties.

\section{Charge Carriers}

The quasi two-dimensional nature of the ET family of organic metals also manifests itself in a variety of galvanomagnetic phenomena, the subjects of recent intensive investigations, which allow us to advance our understanding of some intrinsic electronic properties of these materials. The current carriers in the $a b$-plane have been found to be holes with a very large but as yet inprecisely determined mass for motion in the $c$-direction on the order of $4-5$ times the free electron mass. The carrier concentration $n$ on the order of $10^{21}$ per $\mathrm{cm}^{3}$ found from galvanomagnetic measurements, and coinciding rather well with the value obtained from simple chemical considerations, suggests that one electron is tightly bound to each anion. The concentration is one to one and a half orders of magnitude smaller than those of conventional metals. Together with such small concen- trations of carriers, the experimentally determined Fermi velocity of the carriers in the $a b$-plane of $10^{7} \mathrm{~cm} \mathrm{~s}^{-1}$ is an order of magnitude less than that for conventional metals, and the Fermi energy is on the order of $0.1 \mathrm{eV}$ instead of $3-10 \mathrm{eV}$.

One notes that the structures and the normal electronic properties of the twodimensional organic superconductors are in many respects similar to the corresponding features of the high-temperature oxide superconductors. The latter also have layered crystal structures and are rather "bad" metals from the point of view of the concentration of current carriers.

\section{Superconducting Properties}

It has been mentioned that the maximum superconducting transition temperature $T_{c}$ of the organic superconductors has increased from $1 \mathrm{~K}$ to $12.5 \mathrm{~K}$ in the 10 years since their discovery. Today's maximum temperature cannot yet be referred to as a "high" one since the theoretically predicted lower limit is approximately $30 \mathrm{~K}$ (we should recall that $\mathrm{BiPbBaO}$, the first oxide superconductor family to be discovered, has a $T_{c}$ near $13 \mathrm{~K}$ as compared with recently discovered families that have $T_{c}^{\prime}$ 's approaching $130 \mathrm{~K}$ ).

\section{Type II superconductivity}

All organic superconductors are of Type II as there exist two critical fields $H_{\mathrm{c} 1}$ and $H_{\mathrm{c} 2}$, at which the magnetic field begins to penetrate into the material in the form of Abricosov vortices, and at which superconductivity disappears. The type of superconductivity is determined by the ratio $\lambda / \xi$ of the penetration depth $\lambda$ and the coherence length $\xi$ : for Type II superconductivity 
the ratio is $>1 / \sqrt{2}$. The penetration distance, the depth to which a small magnetic field penetrates into a superconducting metal, is given by $m c^{2} / 4 \pi n e^{2}$ and is large for a large electron mass $m$ and a small electron concentration $n$ ( $c$ is the velocity of light and $e$ the electron charge). The coherence length $\xi \approx 0.2$ $\left(\hbar v_{F} / k_{B} T_{c}\right)$ is roughly the size of the Cooper electron pairs which arise in a metal after the superconducting transition and form the superconducting electron liquid ( $h$ and $k_{\mathrm{B}}$ are the Planck and Boltzmann constants, respectively). It is small for a large $T_{c}$ and a small Fermi velocity $v_{F}$. One thus expects $\lambda$ to be large and $\xi$ small in poorly conducting "metals" with rather high transition temperatures such as organic or oxide superconductors, so these materials should be Type II superconductors characterized by $\lambda / \xi>1 / 2$.

There have been only few reported attempts to determine $\lambda$ in the quasi two-dimensional organic superconductors. They give values on the order of $10^{3} \AA$ in the $a b$-plane. The coherence length $\xi_{a b}$ in the same plane has been found to be on the order of $10^{2} \AA$, and $\xi_{\mathrm{ac}} \approx \xi_{\mathrm{bc}} \approx 10 \AA$. These values are fully consistent with the normal state parameters described above.

\section{Non-magnetic impurities}

At present, the nature of the interactions leading to the formation of superconducting pairs in organic superconductors remains unknown although ordinary BCS superconductivity with the usual phonon mediated pairing can still be considered as a likely interpretation. However, more exotic pairing mechanisms have been proposed, in parti- cular pairing mediated by spin excitations instead of phonons.

The only feature which definitely points to an exceptional property is the extremely strong dependence of transition temperatures on the concentration of non-magnetic impurities. The most prominent demonstration of this sensitivity is shown by the $\beta$-(ET) $I_{3}$ salt. Its two phases, $\beta_{L}$ and $\beta_{H}$, have transition temperatures of 1.5 and $8 \mathrm{~K}$ respectively, differing by more than a factor of five. Measurements of several normal state properties, including paramagnetic susceptibility and the proton magnetic resonance relaxation rate, nevertheless show that the electron systems in these two phases have very similar characteristics. This is also confirmed by the observation of approximately the same values for the gradients of the $\rho$ versus $T^{2}$ plots for the two phases, confirming that the strength of the electron-electron interaction remains roughly constant. The only difference is the presence in the $\beta_{L}$-phase of an additional electron scattering mechanism, absent in the $\beta_{H^{-}}$-phase, that is associated with partial disordering of the crystal lattice. The most notable manifestation of this disordering is a residual resistance $\rho_{0}$ some 20-30 times larger in $\beta_{L}$ than in $\beta_{H}$. It is well known that scattering on such non-magnetic defects formed by lattice disordering does not affect conventional superconductivity. However, the high sensitivity of the superconducting transition temperature to the presence of non-magnetic impurities (which is a characteristic feature of all known organic superconductors) as well as the observation that the nuclear spin-lattice relaxation rate of (TMTSF) 2
$\mathrm{ClO}_{4}$ follows a power law temperature dependence in the superconducting phase point to unusual mechanisms for superconductivity.

\section{Conclusions}

This brief review of organic superconductors demonstrates that truely high temperature superconductivity was found down a different path. Does this mean that all our efforts have turned out to be useless? I think not. The presence in solid state physics of the novel objects, namely highly anisotropic organic metals and superconductors, has resulted in the observation of a number of new phenomena, such as the Peierls instability, charge and spin density waves, phonon-assisted electron localization and so on. All this has contributed to the clarification of some old concepts concerning both the normal and superconducting state in metals, and to the formulation of some new ones. Some of these concepts have turned out to be useful for understanding, in particular, various properties of the high- $T_{c}$ oxide superconductors, which also have layered crystal structures and many other features in common with their organic counterparts.

Finally, and perhaps most important of all, I believe that we have not heard the last word on organic superconductivity: we should anticipate the appearance of new materials, new properties and new ideas.

\section{REFERENCES}

[1] Ribault M. et al., J. Physique Lett. 41 (1980) L607.

[2] Jérome D. and Bechgaard K., Europhys. News 14 (1983) 5, 7.

[3] Jérome D., Phys. Bull. 37 (1986) 171.

\title{
Science and Technology of Conducting Polymers
}

\author{
EIW-6, Europhysics Industrial Workshop
}

Lofthus, Norway, 28-31 May 1990

The sixth in the series of Europhysics Industrial Workshops on the science and technology of conducting polymers was held in Lofthus, Norway on 28-31 May 1990. It was organized by W.R. Salaneck, Linköping University, Sweden, D.T. Clark, I.C.I., England, and E.J. Samuelsen, Trondheim University, Norway and was attended by almost 60 participants, with approximately equal numbers from industry and academia. The venue, the Hotel Ullensvang located on the Hardanger Fjord about $120 \mathrm{~km}$ from Bergen, turned out to be somewhat
The Hardanger Fjord from the Hotel Ullensvang where EIW- 6 was held.

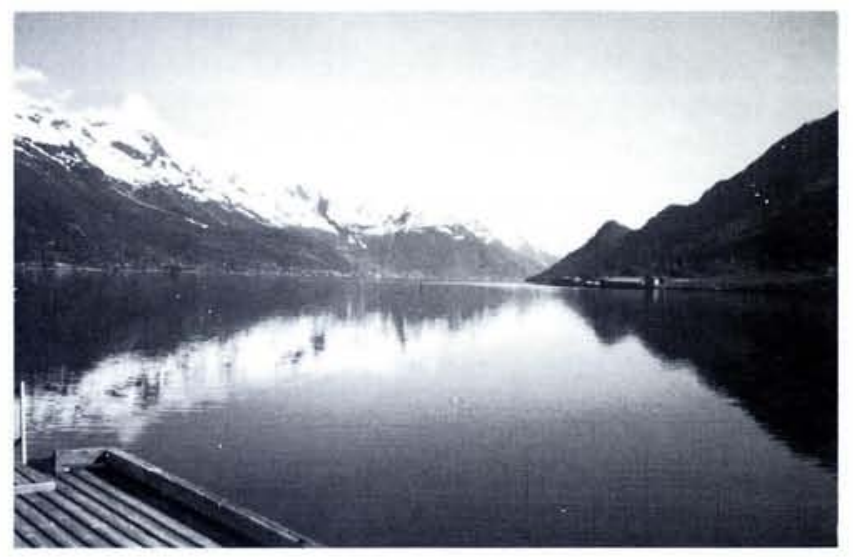

difficult to reach owing strikes at the local airlines, but this merely contributed to the excitement of the event.

The scientific program consisted of $21 / 2$ days of oral presentations and an evening poster session. Of the 17 speakers, 6 were from industry and 11 from academia. Tutorial lectures treating the basic physics and chemistry of conjugated polymers, both undoped as well as doped to a state of high electrical conductivity ("conducting" polymers), comprised about $1 / 3$ of the presenta- 